November 6, 2018

SLAC-PUB-8008

SU-ITP-98/62

hep-ph/9811353

\title{
New origin for approximate symmetries from distant breaking in extra dimensions
}

\author{
Nima Arkani-Hamed ${ }^{a}$ and Savas Dimopoulos ${ }^{b}$ \\ a SLAC, Stanford, California 94309, USA \\ ${ }^{b}$ Physics Dept., Stanford University, Stanford, California 94309, USA
}

\begin{abstract}
The recently proposed theories with $\mathrm{TeV}$-scale quantum gravity do not have the usual ultraviolet desert between $\sim 10^{3}-10^{19} \mathrm{GeV}$ where effective field theory ideas apply. Consequently, the success of the desert in explaining approximate symmetries is lost, and theories of flavor, neutrino masses, proton longevity or supersymmetry breaking, lose their usual habitat. In this paper we show that these ideas can find a new home in an infrared desert: the large space in the extra dimensions. The main idea is that symmetries are primordially exact on our brane, but are broken at $O(1)$ on distant branes. This breaking is communicated to us in a distance-suppressed way by bulk messengers. We illustrate these ideas in a number of settings: 1) We construct theories for the fermion mass hierarchy which avoid problems with large flavor-changing neutral currents. 2) We re-iterate that proton stability can arise if baryon number is gauged in the bulk. 3) We study limits on light gauge fields and scalars in the bulk coming from rare decays, astrophysics and cosmology. 4) We remark that the same ideas can be used to explain small neutrino masses, as well as hierarchical supersymmetry breaking. 5) We construct a theory with bulk technicolor, avoiding the difficulties with extended technicolor. There are also a number of interesting experimental signals of these ideas: 1) Attractive or repulsive, isotope dependent sub-millimeter forces $\sim 10^{6}$ times gravitational strength, from the exchange of light bulk particles. 2) Novel Higgs decays to light generation fermions plus bulk scalars. 3) Collider production of bulk vector and scalar fields, leading to $\gamma$ or jet+ missing energy signals as in the case of bulk graviton production, with comparable or larger rates.
\end{abstract}




\section{Life without the desert}

The standard paradigm of particle physics dates back to the advent of grand unified theories [1] or perhaps even further back to Fermi's theory of beta decay. Its premise is that there are two fundamental scales -the weak and Planck masses- separated by a large "desert". The existence of the desert plays a fundamental role in formulating and solving problems in particle physics. Examples include the physics of flavor, neutrino masses and of unification. Much of the physics of the early universe takes place when the temperature of the universe is in the desert. The very hierarchy problem is simply the statement of the large size of the desert. This suggested a new proposal for solving the hierarchy problem simply postulating that the desert does not exist: namely that the fundamental scale of gravity is in fact identical to the weak interaction scale $\sim \mathrm{TeV}$ [2, 3, 4]. In this new paradigm, the observed weakness of gravity at long distances is due the existence of new sub-millimeter spatial dimensions into which gravity spreads. The standard model fields are localized to a $(3+1)$-dimensional wall or "3-brane". The hierarchy problem becomes isomorphic to the problem of the large size of the extra dimensions. Some ideas for stabilizing large dimensions have been explored in [5, 6].

Whereas the absence of a desert may allow for a novel approach to the hierarchy problem, it also deprives us from all mechanisms whose existence relied on the desert. This includes baryon stability, naturalness of approximate lepton number conservation and neutrino masses, as well as approximate neutral flavor conservation which are some of the successes of the nonsupersymmetric standard model 冈. In addition there are phenomena and mechanisms in extensions of the standard model such as the supersymmetric gauge coupling constant unification, electroweak breaking in technicolor or susy, supersymmetry breaking as well as models of flavor which largely relies on the existence of the desert. It is the purpose of this paper to find new and natural mechanisms to account for some of these phenomena within the new framework of theories without a desert.

While we have been deprived of the desert in short-distance scales between the weak and Planck scales, we have gained the large space in the extra

*Note that the naturallness of these successes of the standard model is lost in any $\mathrm{TeV}$ extension of the standard model, including low energy supersymmetry 
dimensions where new mechanisms can reside. In this paper we present ideas for generating natural flavor hierarchies of quark and lepton masses and show that they do not lead to unacceptably large flavor violations. The same generic mechanism can be used to understand the approximate conservation of baryon and lepton number. We also note in passing that in theories with a high fundamental Planck scale, these same ideas can furnish a simple mechanism for dynamical supersymmetry breaking.

The basic idea which makes all of this possible is that symmetries which are primordially good on our brane maybe broken on other distant branes by a large $O(1)$ amount. The information of this breaking is transmitted to us by messenger fields living in the bulk, and is suppressed by the distance between our brane and the others. If the messengers are massive, an exponential suppression of the symmetry breaking on our wall results, while even if they are very light a power suppression is possible.

The case of light messengers is particularly interesting since they can macroscopic forces which may be detected by the upcoming sub-millimeter tests of gravitational strength forces [7]. Indeed, in many cases, the forces are expected to be at least $10^{6}$ times gravitational strength. Of course, the constraints on very light messengers coming from rare decays, astrophysics and cosmology must also be considered. We find that there is still a significant region of experimentally viable parameter space where the dramatic predictions of $10^{6}$ times gravity sub-millimeter forces are possible.

If the bulk messengers are heavier than a few $\mathrm{GeV}$, all the previous constraints disappear, as does the signal for sub-millimeter experiments. On the other hand, in some cases, novel Higgs decays to light SM generations and bulk modes are possible, which can be comparable or even dominate over the usual decay channels. Furthermore, the production of bulk gauge fields or scalars can be probed via $\gamma$ or jet + missing energy signals much as in the case of bulk graviton production, but with a much larger rate.

Finally, as a somewhat different illustration of new possibilities for interesting phenomenology from extra dimensions, we show that electroweak symmetry breaking on our wall does not require a fundamental Higgs field, but can be induces via a technicolor gauge force in the bulk triggering a technifermion condensate on our wall. This can be combined with the other ideas for generating flavor, thereby avoiding the usual problems with extended technicolor. 


\section{Yukawas from distant flavor breaking}

A popular mechanism for explaining the smallness of the observed Yukawa couplings invokes a flavor symmetry $G_{F}$ under which the SM generations, and perhaps the Higgs, are charged. The representations under $G_{F}$ are chosen such that none of the light generation Yukawa couplings $f f^{c} H$ are neutral under $G_{F}$, but the large Yukawa couplings are invariant. In addition, there are a certain number of "flavon" fields $\phi$, charged under $G_{F}$, which are assumed to acquire vevs from some dynamics thereby breaking $G_{F}$. In the effective theory beneath some scale $M_{F}$, we expect $G_{F}$ invariant higher dimension operators of the generic form

$$
\mathcal{O} \sim \lambda \frac{\phi^{n}}{M_{F}^{n}} f f^{c} H
$$

with $\lambda \sim O(1)$ to be present in the theory. If $M_{F}$ is close to the string scale $M_{s}$ these operators can be generated by quantum gravity effects. If $M_{F} \ll M_{s}$ these operators can be generated by integrating out heavy fields of mass $\sim M_{F}$ which carry flavor charge (the Froggatt-Nielsen mechanism [8]). Regardless, if we assume that $\epsilon \sim\langle\phi\rangle / M_{F}$ is small, the small Yukawa couplings can be understood as powers in the small parameter $\epsilon$. Of course, this does not constitute a full understanding of the smallness of the fermion masses until the mechanism for triggering a vev for $\phi$ smaller than $M_{F}$ is presented.

In this section, we will show how in the presence of extra dimensions populated with multiple 3-branes, we can take advantage of flavor symmetries to explain small Yukawa couplings without needing a small flavor breaking scale $\langle\phi\rangle \ll M_{F}$. In fact, we will assume that the flavor symmetry is badly broken at the string scale $\langle\phi\rangle \sim M_{s}$. However, the flavons $\phi$ are taken to live on a different 3-brane than ours. Therefore, some "messenger" needs to communicate the information of flavor breaking to our 3-brane. It is easy to see that gravity in the bulk cannot generate any $G_{F}$ violating operator involving wall fields. The reason is simple: gravity is not charged under $G_{F}$. Any operator generated by gravitational exchange will be of the form $O_{\text {ourwall }} O_{\text {otherwall }}$ where each $O$ is individually $G_{F}$ symmetric. This generalises to an intuitively obvious statement: no $G_{F}$ violating operators can be induced on our wall unless there are bulk modes charged under $G_{F}$. If this messenger is massive, the resulting coefficient $\lambda$ in eqn.(11) will be expo- 
nentially suppressed by the distance between the 3-branes, while even if the messenger is massless, a power suppression of $\lambda$ is possible.

We note here that the generic mechanism for obtaining small Yukawas presented below works for any value of $M_{s}$, not just for $M_{s}$ near the $\mathrm{TeV}$ scale. Nonetheless, we will mostly be interested in the latter case, where the correlation between generating flavor while avoiding flavor-changing problems is particularly challenging and interesting. It is easy to understand the idea in the following simple toy example. Suppose that there is an abelian (continuous or discrete) $G_{F}$ such that the electron Yukawa coupling $L_{1} H E_{1}^{c}$ carries flavor charge 1 , and that there is a flavon $\phi$ with charge -1 , which acquires a string scale vev $\langle\phi\rangle \sim M_{s}$, but which lives on a different 3-brane parallel to ours, a transverse distance $r$ away from our 3-brane. In order to communicate the information of $G_{F}$ breaking between the 3-branes, we have a scalar field $\chi$ of mass $m_{\chi}$, with charge -1 under $G_{F}$. For definiteness, let the co-ordinates of our 3 -brane be $\left(x, y^{a}=0\right)$ while those of the other brane are $\left(x^{\prime}, y^{a}=y_{*}^{a}\right)$, where $a=1, \cdots, n$ runs over the extra "large" dimensions and $\left|y_{*}\right|=r$ is the transverse distance between the branes. We expect all possible local operators consistent with symmeties to be generated by physics above the string scale $M_{s}$, in particular we can have

$$
\mathcal{L} \supset \int_{\mathrm{us}} d^{4} x L H E^{c}(x) \chi\left(x, y^{a}=0\right)+\int_{\text {other }} d^{4} x^{\prime} \phi\left(x^{\prime}\right) \chi^{*}\left(x^{\prime}, y^{a}=y_{*}^{a}\right)
$$

where we have set $M_{s}=1$. For simplicity, let us for the moment ignore possible non-linear self-interactions of the $\chi$ field with itself in the bulk. Then, the non-zero vev if $\phi$ acts as a linear source, "shining" the $\chi$ field. The profile of the $\chi$ field created by this source is easily obtained. Since $\langle\phi\rangle$ is $x^{\prime}$ independent, the value $\langle\chi\rangle$ only depends on the transverse distance from the other brane, and is given by the Yukawa potential in the $n$ transverse dimensions:

$$
\langle\chi\rangle(x, y)=\langle\phi\rangle \times \Delta_{n}\left(\left|y-y_{*}\right|\right)
$$

where

$$
\Delta_{n}(y)=\left(-\left(\partial^{2}\right)_{n}+m_{\chi}^{2}\right)^{-1}(y)=\int d^{n} \kappa e^{i \kappa y} \frac{1}{\kappa^{2}+m_{\chi}^{2}} .
$$

More explicitly, we have

$$
\begin{aligned}
\langle\chi\rangle(x, y) & =\int d^{4} x^{\prime}\left(\left(\partial^{2}\right)_{4}-\left(\partial^{2}\right)_{n}+m_{\chi}^{2}\right)^{-1}\left(x-x^{\prime}, y-y_{*}\right)\langle\phi\rangle\left(x^{\prime}\right) \\
& =\langle\phi\rangle \times\left(-\left(\partial^{2}\right)_{n}+m_{\chi}^{2}\right)^{-1}\left(y-y_{*}\right) .
\end{aligned}
$$


Note that we should actually be using the propagator appropriate to the compactified space (i.e. the integral over $\kappa$ in eqn.(4) should be replaced with a sum over KK modes), however, the difference is negligible as long as $\left|y-y_{*}\right|$ is less than $r_{n}$, the size of the extra dimensions.

There is now a Yukawa coupling generated from the "shined" value of $\langle\chi\rangle$ on our wall

$$
\mathcal{O}=\int d^{4} x\langle\chi\rangle(x, y=0) L H E^{c}(x)=\int d^{4} x\langle\phi\rangle \Delta_{n}(r) L H E^{c}(x) .
$$

Recall that for $n>2$, we have

$$
\begin{aligned}
\Delta_{n}(r) & \sim \frac{1}{r^{n-2}}\left(r m_{\chi} \ll 1\right) \\
& \sim \frac{e^{-m_{\chi} r}}{r^{n-2}}\left(r m_{\chi} \gg 1\right)
\end{aligned}
$$

while for $n=2$

$$
\begin{aligned}
\Delta_{n}(r) & \sim-\log \left(r m_{\chi}\right)\left(r m_{\chi} \ll 1\right) \\
& \sim \frac{e^{-m_{\chi} r}}{\sqrt{m_{\chi} r}}\left(r m_{\chi} \gg 1\right) .
\end{aligned}
$$

Thus, we get the expected $e^{-m r}$ suppression of the electron Yukawa coupling if the branes are separated by more than $m_{\chi}^{-1}$, while for $n>2$, we still get the geometrical power suppression $\sim r^{2-n}$ even for $m_{\chi}=0$. Of course, we can not have a massless scalar coupled to the ordinary stable matter because of conflicts with the tests of the equivalence principle. In any case, we do not expect scalars to stay light unless protected by some symmetry. Interestingly, if there is SUSY in the bulk with SUSY broken on the wall, the scalars can naturally acquire very small masses of $\sim(\mathrm{TeV})^{2} / M_{p l} \sim(\mathrm{mm})^{-1}$, which is precisely in the interesting range for being tested by upcoming submillimeter measurements of gravitational strength forces. The possibility that these experiments might uncover "Yukawa moduli" has previously been discussed in [9], it is amusing that "Yukawa messengers" may also be probed as we will discuss more fully below. Alternately, it could be that there is no SUSY in the bulk and that $\chi$ starts massless but receives radiative corrections which do not quite drag it back up to $M_{s}$ due to couplings or loop factors; it is certainly easy to imagine that $\chi$ is light within one or two orders of 
magnitude of $M_{s}$. In this case, the suppressions in $\Delta_{n}(r)$ may either be exponential or purely geometrical depending on whether the relevant $r$ is bigger or smaller than $m_{\chi}^{-1}$.

Summarizing, in all cases, the smallness of the Yukawa coupling is understood from this very simple picture: the breakings of the flavor symmetries are of $O(1)$ on far away branes, and the smaller intensity of breaking on our branes is simply due either to the geometrical power law fall off (for massless particles and $n>2$ ), or the exponential decay characteristic of massive particles.

Up to now we have been assuming that the other wall is also a 3-brane. We can however consider the possibility that it is an arbitrary $p$-brane, where $p \geq 3$. For $p<3$, different regions of our universe will have different transverse distances to the other brane, and the Yukawa couplings in our universe would vary unacceptably in different regions of the universe. Of course, for $n$ "large" dimensions, the extra $(p-3)$ dimensions of the $p$-brane are not infinite but have sizes $\sim r_{n}$, and we must have $p \leq(n+3)$. The only difference with the case of $p=3$ is that the $p$-brane no longer looks like a point in the $n$ transverse dimensions; rather, it appears as a $p-3$ brane. The value of $\langle\chi\rangle$ "shined" by sources on the $p$-brane will be the potential set up by a $p-3$ brane in the transverse $n$ dimensions, which by the same symmetry arguments used above is the Yukawa potential set up in the $n+3-p$ dimensions transverse to the $p$-brane. Therefore we must replace

$$
\Delta_{n}(r) \rightarrow \Delta_{n+3-p}(r)
$$

in the Yukawa coupling suppressions. For $p>3$, this gives an enhancement of the induced Yukawa coupling over the $p=3$ case, as expected.

Of course, it is also possible that the bulk messengers themselves are not free to propagate in all the extra dimensions, but only $n_{\text {mess }} \leq n$ of them. As long as they can propagate to the other brane, they will still mediate flavor breaking to our wall, and all the previous results hold replacing $n \rightarrow n_{\text {mess }}$.

It is clear that this mechanism can be generalized to explain the small size of the "spurion" $\epsilon \sim \phi / M_{F}$ of any flavor model. We just replace what was formerly considered the small vev of a flavon $\phi$ relative to a higher scale $M_{F}$ by the distance-suppressed vev of a bulk messenger $\chi$ with identical $G_{F}$ quantum numbers as $\phi$. Of course, the question of the origin of fermion mass hierarchy is transmuted in our picture to the question of what determines the 
inter-brane separations. We do not have anything specific to say on this dynamical question. Note however that in the case of exponential suppressions, or even the power law suppressions obtained for $n=6, \sim r^{-4}$, the walls do not need to more than $O(10)$ times the fundamental Planck scale away from each other to cover the observed range of fermion masses, so that at least no large hierarchies in inter-brane separation are needed. One can for instance imagine that there is a moderately large extra dimensions $\sim 10$ times larger than the fundamental scale, and that the different walls are stuck at different orbifold fixed points in this extra dimension. Alternately, there could be dynamical mechanisms where attractive forces between the branes form stable bound systems, much as the planets rotate in stable orbits around the sun.

A different direction for getting small Yukawas is to imagine different SM generations living on different walls. In this scenario, the SM gauge fields must be delocalized and free to propagate at least between the branes where the different generations are trapped. Again, massive modes linking the different branes will give rise to exponentially small Yukawa couplings. Such possibilities have been noted within the context of orbifold compactifications of string theory. This scenario seems to have phenomenological difficulties in the scenario where $M_{s} \sim \mathrm{TeV}$. The reason is that since the cutoff is $\sim 1 \mathrm{TeV}$, if the exponential suppression is to give small Yukawa couplings, we need to have walls perhaps as far as $\sim(100) \mathrm{GeV}^{-1}$. Since the SM gauge fields must be delocalised in the extra dimensions on this scale, these effects would have already been experimentally observed. Also, it seems to not be as easy to avoid flavor-changing problems in this scenario. Of course, these problems can be solved by pushing the higher dimensional Planck scale to sufficiently

but it is clear that this scenario is less safe than one where all the SM fields are localised to an at least $M_{s}^{-1}$ thickness wall.

\section{$3 \quad$ Neutrino masses and SUSY breaking}

It is obvious that the mechanism for generating small parameters presented above is generic and can be used to explain many sorts of small parameters. For instance, we can generate small Majorana neutrino masses if lepton number is broken on far away walls. This idea, together with other intrinsically higher dimensional mechanisms for small neutrino masses will be discussed in [10]. One may perhaps hope to to be able to generate exponential hier- 
archies of scales in this way, perhaps even for the weak-Planck hierarchy. It is certainly easy to generate a Higgs mass parameter exponentially smaller than $M_{P l}$ [11], the difficulty still is that in the absence of SUSY, radiative corrections would make $\sim M_{P l}^{2}$ contributions to the higgs mass ${ }^{2}$. Therefore, some sort of SUSY may still be needed. Nevertheless, we can still construct very simple theories of exponentially small SUSY breaking. Indeed, the most trivial way of breaking SUSY is with a theory of a single chiral field $\phi$ with a linear superpotential $W=\lambda M_{p l}^{2} \phi$. We can generate an exponentially small $\lambda$ in the same way we generated exponentially small Yukawas in the last section, by coupling $\phi$ to a massive field in the bulk which in turn couples to a (SUSY preserving) Planck-scale vev on a different wall. We emphasize that this mechanism is different than breaking SUSY on the other wall and communicating the information to our wall with bulk messengers [12]; here the dynamics on the other wall preserves SUSY, but acts as a source for a massive bulk field whose exponentially suppressed vev on our wall becomes a linear coupling in the superpotential that breaks SUSY directly on our wall.

\section{Constraints and signals from new light states}

\subsection{Distant familons}

So far we have ignored the dynamics on the other branes, other than to assume that they provide an explicit source of $O(1) G_{F}$ violation. If $G_{F}$ is a continuous global symmetry, however, the walls also contain the goldstones of $G_{F}$ breaking, the "familons" with decay constants $\sim M_{s}$. Familons with low decay constants are a disaster because they can be produced in dangerous flavor-changing transitions such as $K \rightarrow \pi+$ familon, which typically

force the decay constants above $\sim 10^{12} \mathrm{GeV}$. One might hope that since in our case the familons live on different branes, they have suppressed couplings and are therefore harder to produce. However, at low energies, the distance between the walls can not be resolved; the couplings of the familons to the SM fields are then dictated as usual by the non-linear realization of $G_{F}$. More explicitly, if the source for $\chi$ on the other wall depends on massless fields as $\langle\phi\rangle \rightarrow \exp \left(i \pi^{a} T^{a}\right)\langle\phi\rangle$, the "shined" vev of $\chi$, and therefore the Yukawa couplings on our wall, also depends on these massless fields precisely as dictated by the non-linear realization of the symmetry. Of course this is not a problem 
if we wish to consider theories with high fundamental scales $M_{s} \geq 10^{12} \mathrm{GeV}$, but for theories with much lower $M_{s}$, this issue needs to be addressed. One's first thought is to gauge the symmetry in order to eat the familon. This does not work because the gauge boson would then necessarily be a bulk field with very weak coupling to wall states, only picking up an $\sim M_{s}^{2} / M_{P l}$ mass from $\sim M_{s}$ breaking on a three-brane [4]. For $M_{s} \sim \mathrm{TeV}$ this is so light that the familon (longitudinal component of the gauge boson) can still be produced in decays. The simplest solution is to have only discrete flavor symmetries, and therefore no light states on the other walls. Note that in the usual theories of flavor, simply decreeing that the true flavor symmetry is discrete does not guarantee the absence of light familons, since the renormalizable interactions of the theory typically admit accidental continuous symmetries which are then spontaneously broken yielding pseudo-goldstone bosons. Higher dimension operators preserving the discrete symmetry but violating the accidental continuous symmetry will give mass to the pseudo-goldstones, but these will be suppressed by a power of the ratio of the flavor breaking scale to that of the higher dimension operators. This need not be the case in our scenario, since we are maximally breaking the flavor symmetry at the fundamental scale $M_{s}$, so that the higher dimension operators distinguishing the continuous from discrete symmetries are unsuppressed. Note that this effectively allows us to use continuous $G_{F}$ : one can always imagine that we are really considering an arbitrarily large discrete subgroup of $G_{F}$, to avoid the problem with familons on the other wall, while keeping the same predictions for flavor-violations on our wall to arbitrarily high accuracy. From the point of view of the low-energy theory, there is only explicit violations of $G_{F}$ on the other walls, with no other light states to indicate spontaneous breaking.

\subsection{Light messengers of flavor breaking}

In the case where $\chi$ is very light, perhaps only getting $\mathrm{a} \sim(1 \mathrm{~mm})^{-1} \sim 10^{-3}$ $\mathrm{eV}$ mass, we have the interesting possibility of observable sub-millimeter forces mediated by $\chi$ exchange. Recall that the couplings of $\chi$ to SM fields is of the form

$$
\mathcal{L} \sim \int d^{4} x f_{i} f_{j^{c}}^{c} H(x) \frac{\chi_{i j^{c}}(x, y=0)}{M_{s}^{(n+2) / 2}}
$$

Expanding

$$
\chi(x, y=0)=\langle\chi\rangle(x, y=0)+\chi^{\prime}(x, y=0)
$$


it is readily apparent $\chi^{\prime}$ can in general have both flavor-changing and flavorviolating couplings. For $n>2$, all the KK excitations of $\chi^{\prime}$ are too heavy to be relevant to sub-millimeter force experiments, so only the coupling of the zero mode is needed. Since $\chi^{\prime}$ is a bulk field, this coupling is suppressed by the value of the wavefunction of the zero mode on the wall $\sim 1 / \sqrt{V_{n}}$. More formally, $\chi^{\prime}$ is Fourier expanded as

$$
\chi^{\prime}(x, y=0)=\sum_{k_{1}, \cdots, k_{n}} \frac{1}{\sqrt{V_{n}}} \chi^{k_{1}, \cdots, k_{n}}(x)
$$

The long range force that is generated from the Yukawa coupling to the zero mode

$$
\rho f f^{c} \chi^{0}, \rho=\frac{v}{M_{p l}} \sim 10^{-16}
$$

where we have used the relation $M_{p l}^{2} \sim M_{s}^{n+2} V_{n}$. Note that this Yukawa coupling is both $n$ and $M_{s}$ independent. While $\rho$ seems miniscule, it dominates gravity by a factor of $\sim 10^{6}$ at distances shorter than the $1 / m_{\chi}$. For example, the ratio of $\chi$ exchange force to gravity for nucleons is

$$
F_{\chi}: F_{\text {grav }}=\rho^{2}: G_{N} m_{\text {nucleon }}^{2} \sim 10^{6}
$$

In this respect the $\chi^{\prime}$ field mediates a force of the same order of magnitude as gauge bosons in the bulk coupled to a linear combination of $B, L$ as discussed in [4] and more fully discussed in Section 7. There is one difference: the effective 4-dimensional gauge coupling is $g \sim M_{s} / M_{p l}$, and grows as $M_{s}$ is pushed above a TeV. On the other hand, as remarked above, the strength of the Yukawa force is independent of $M_{s}$. This spectacular signature of sub$\mathrm{mm}$ forces a million times stronger than gravity only depends on having $\chi^{\prime} s$ sufficiently light that their Compton wavelength falls in the range $\sim 1 \mu m-$ $1 \mathrm{~mm}$ soon to be probed by experiment. Furthermore, the force mediated by $\chi$ exchange can be distinguished from similar size bulk gauge field induced forces, since the latter is repulsive while the former is attractive .

It is instructive to compare this "Yukawa messenger" force with the "Yukawa modulus" force discussed in [9]. In [9], supersymmetric theories were considered with a continuous global flavor symmetry $G_{F}$ spontaneously broken at the usual 4-d Planck scale. The "Yukawa moduli" are just the goldstones of $G_{F}$ breaking. In a supersymmetric theory, there is a full goldstone chiral multiplet $\Phi^{a}$ for each broken generator $X^{a}$ of $G_{F}$. The complex 
scalar $\phi^{a}=\sigma^{a}+i \pi^{a}$ includes the usual goldstone field $\left(\pi^{a}\right)$ together with a scalar partner $\sigma^{a}$ whose mass is only protected by unbroken SUSY. If SUSY is broken at very low energies, these fields can pick up a small mass $\sim(\mathrm{TeV})^{2} / M_{p l} \sim(1 \mathrm{~mm})^{-1}$ and can mediate interesting sub-mm forces. The linear couplings of $\phi^{a}$ are fixed by the non-linear realisation of $G_{F}$ to be given by

$$
W \supset \phi^{a} \delta_{X^{a}}\left(\lambda^{i j^{c}} f_{i} f_{j^{c}}^{c}\right)
$$

where $\delta_{X^{a}}(f)$ denotes the first order variation of $f$ with respect to the broken generators $X^{a}$ of $G_{F}$. The crucial point is that the couplings of $\phi^{a}$ are suppressed by SM particle masses. Of course this is because all couplings must vanish in the limit where the SM chiral symmetries are unbroken. The force mediated by the scalar partners of the goldstones are therefore truly gravitational in strength. By contrast, the force mediated by the "Yukawa messengers" are present even if $\chi$ does not acquire a vev on our wall, and the strength of the force is enhanced by the $\sim 10^{6}$ factor we found in the previous paragraph.

Given the obvious interest in these signals, and their crucial reliance on a light $\sim(\mathrm{mm})^{-1}$ mass, it is important to insure that such light $\chi$ 's are not excluded on other grounds. As we have remarked, the sub-mm force is actually independent of the value of the fundamental scale $M_{s}$, while the dangerous production of bulk $\chi$ modes is suppressed by powers of $M_{s}$. Therefore, we can always take $M_{s}$ high enough to avoid phenomenological problems while keeping the sub-mm signal. What we are interested here is how low $M_{s}$ can be, and in particular whether $M_{s} \sim \mathrm{TeV}$ is allowed. Indeed, we will find quite significant constraints even on the flavor conserving interactions of the $\chi$ fields from astrophysics and cosmology, while the flavor-violating couplings are even more severely from rare decays. Nevertheless, $M_{s}>10^{6} \mathrm{TeV}$ satisfies all constraints in all cases, while $M_{s} \sim \mathrm{TeV}$ is allowed for the cases $n \geq 4$.

Let us begin with the flavor-conserving $\chi$ interactions, considering the bounds from astrophysics and cosmology which result from the overproduction of bulk $\chi$ modes. Let us first perform the analogue of the dimensional analysis done in [4] to determine the scaling of the rate for $\chi$ production with the temperature $T$ of wall states. From the couplings given in eqn.(10) this 
rate is determined by dimensional analysis to be

$$
\text { rate for } \chi \text { prod. } \sim \frac{v^{2} T^{n-2}}{M_{s}^{n+2}}
$$

where $v \sim 175 \mathrm{GeV}$ is the Higgs vev. Notice that just like gravitons, this rate grows softer in the infrared as $n$ increases, as is to be expected for a bulk mode. However, it is not quite as soft as the rate for graviton production

$$
\text { rate for grav. prod. } \sim \frac{T^{n}}{M_{s}^{n+2}}
$$

Therefore, the constraints on this scenario are stronger than the corresponding ones for gravitons. In fact, for $M_{s} \sim \mathrm{TeV}$, we can roughly see that the constraints on $\chi$ for $n$ extra dimensions are the same as for gravitons with $n-2$ extra dimensions. For gravitons, the constraints for $n=2$ from the Supernova forces $M_{s} \sim 30 \mathrm{TeV}$, while $M_{s} \sim \mathrm{TeV}$ was safe for $n>2$. Therefore, the constraints from $\chi$ overproduction in the SN rule out the cases $n=2,3$, force $M_{s} \geq 30 \mathrm{TeV}$ for $n=4$ and can have $M_{s} \sim \mathrm{TeV}$ for $n \geq 5$.

Similarly, the bounds on the "normalcy" temperature $T_{*}$ in the early universe coming from $\chi$ evaporation, overcooling the universe, are the same as gravitons with $n$ replaced with $n-2$, once again ruling out $n=2,3$, while $n \geq 4$ has $T_{*} \geq 10 \mathrm{MeV}$, leaving nucleosynthesis safely unaltered. The strongest cosmological constraint on gravitons arose because their lifetime for decaying back into wall states exceeded the age of the universe, so that overclosure and the distortion of the background gamma ray spectrum from their decays had to be considered. In our case, however, these bounds do not apply. The reason is that, since the $\chi$ fields are more strongly coupled, they decay more quickly. Indeed, the width for a given KK excitation of $\chi$, produced at temperature $T$ (and therefore having a mass from the 4-d point of view $\sim T$ ) to decay to SM states on the wall is

$$
\Gamma \sim \frac{v^{2}}{M_{p l}^{2}} T
$$

which gives a lifetime for $\chi$ ranging between 10 to 10,000 years for $T \sim 1 \mathrm{GeV}$ - $1 \mathrm{MeV}$. Since these times are before recombination, the decay products are harmless and rethermalise. There is also no worry about the decay products destroying weakly bound states like deuterium. Their lifetime is long enough 
that, by the time they decay, the universe is so dilute that the decay products can not interact with enough deuterons to destroy significant numbers of them.

Summarizing, the constraints on the flavor-conserving $\chi$ interactions rule out $n=2,3$ (at least for $M_{s} \sim \mathrm{TeV}$ ), while they force $M_{s} \sim 30 \mathrm{TeV}$ for $n=4$ and are fine for $M_{s} \sim \mathrm{TeV}$ for $n \geq 4$.

Far more important constraints result from the flavor-violating couplings of $\chi$. Most worrisome are dangerous processes like $K \rightarrow \pi+\chi_{s d}^{\prime}$ or $\mu \rightarrow$ $e+\chi_{\mu e}^{\prime}$. Consider the decay $K \rightarrow \pi+\chi^{\prime}$. By the dimensional analysis familiar from [四], we have

$$
\Gamma\left(K \rightarrow \pi+\chi^{\prime}\right) \sim \frac{v^{2} m_{K}^{n+1}}{M_{s}^{n+2}}
$$

where $v \sim 250 \mathrm{GeV}$ is the Higgs vev. Requiring that the branching ratio be less than the experimental limit of $\sim 7 \times 10^{-9}$ puts a lower bound on $M_{s}$ :

$$
M_{s}>10^{\frac{31-3 n}{n+2}} \mathrm{TeV}
$$

which ranges from $\sim 10^{6} \mathrm{TeV}$ for $n=3$ to $\sim 30 \mathrm{TeV}$ for $n=6$. We see that if we wish to have $M_{s} \sim \mathrm{TeV}$, the mass of the $\chi$ field coupling to strange and down squarks must be pushed above $\sim 1 \mathrm{GeV}$ in order not to be produced in Kaon decays. The branching ratio for $\mu \rightarrow e+$ familon is $<1 \times 10^{-10}$, and an identical computation for $\mu \rightarrow e+\chi_{\mu e}^{\prime}$ yields the bound

$$
M_{s}>10^{\frac{26-4 n}{n+2}} \mathrm{TeV}
$$

which ranged from $\sim 3000 \mathrm{TeV}$ for $n=3$ to $\sim 2 \mathrm{TeV}$ for $n=6$. It is more conceivable that such a light $\chi$ could couple to the lepton sector for $\mathrm{TeV}$ scale $M_{s}$ in this case, and sub-millimeter "Yukawa messenger" signals in the lepton sector are still consistent with $M_{s} \sim \mathrm{TeV}$.

\section{$5 \quad$ Novel Higgs decays}

All the constraints from rare decays, astrophysics and cosmology disappear if the $\chi$ fields are heavier than a few GeV. On the other hand, if they are this heavy, they will not give rise to signals for the upcoming sub-millimeter 
force experiments. On the other hand, if the $\chi$ 's are lighter than any physical Higgs modes, they can be produced in novel Higgs decays with significant rates. It is normally believed that the Higgs couples most strongly to the heaviest generations, since they have the largest Yukawa coupling. Therefore, the dominant decay mode for the neutral Higgs with mass $m_{H^{0}}<2 m_{t}$ is to $b \bar{b}$, with width

$$
\Gamma\left(H^{0} \rightarrow b \bar{b}\right)=\frac{\lambda_{b}^{2}}{16 \pi} m_{H}
$$

However, in our scenario, the Yukawa couplings are the vevs of $\chi$ on our wall, and expanding as usual $\chi(x, y=0)=\langle\chi\rangle(x, y=0)+\chi^{\prime}(x, y=0)$, the relevant interactions of $H$ with the SM fermions and $\chi^{\prime}$ are given by

$$
\mathcal{L}=\int d^{4} x \lambda_{i j^{c}} f^{i} f^{j^{c}} H+\kappa \frac{\chi_{i j^{c}}^{\prime}}{M_{s}^{(n+2) / 2}}(x, y=0) f^{i} f^{j^{c}} H
$$

where we have restored the dependence on the fundamental scale $M_{s}$ and $\kappa$ is a dimensionless coupling, $\lambda_{i j^{c}}=\kappa\left\langle\chi_{i j^{c}}\right\rangle$. We see that while the coupling of $H$ to $f^{i} f^{j^{c}}$ alone is suppressed by the Yukawa coupling for lighter generations, the couplings of $H$ to $f f^{c} \chi^{\prime}$ are are not suppressed by small Yukawas but only by the scale $M_{s}$. The width for $H \rightarrow f f^{c} \chi$, where $\chi$ escapes into the bulk as missing energy, is easily estimated

$$
\Gamma\left(H^{0} \rightarrow f f^{c} \chi\right) \sim \kappa^{2} \frac{m_{H}^{n+3}}{16 \pi M_{s}^{n+2}}
$$

This gives a branching fraction relative to the usual $b \bar{b}$ mode of

$$
\frac{\Gamma\left(H^{0} \rightarrow f f^{c} \chi\right)}{\Gamma\left(H^{0} \rightarrow b \bar{b}\right)} \sim \frac{\kappa^{2}}{\lambda_{b}^{2}}\left(\frac{m_{H}}{M_{s}}\right)^{n+2} .
$$

For relatively low $n=2,3$, this mode can have a significant branching fraction for $M_{s} / \kappa \sim 1 \mathrm{TeV}$. For instance, we can have $O(1)$ branching fractions for $M_{s} / \kappa \sim 1 \mathrm{TeV}$ for $n=2, m_{H} \sim 100 \mathrm{GeV}$ or $n=3, m_{H} \sim 200 \mathrm{GeV}$. Furthermore, the decays $H^{0} \rightarrow e(\mu)^{+} e(\mu)^{-}+(\chi=$ missing energy) are very clean in a hadronic environment, where the $b \bar{b}$ signal is not. Indeed, recall that the usual signal for Higgs production at Hadron colliders is via $H^{0} \rightarrow \gamma \gamma$ which typically has a tiny branching fraction $\sim 10^{-3} \rightarrow 10^{-4}$. These new Higgs signals have enormous branching fractions in comparison and may be 
the dominant discovery mode for the Higgs at hadron colliders, although a more detailed analysis is clearly needed. Since the branching ratio for decaying to bulk modes increases with the mass of the decaying particles, even larger widths are possible for the charged Higgs modes in two Higgs doublet models, for instance $H^{+} \rightarrow e^{+}$or $\mu^{+}+\chi$. Of course the usual decay to top+bottom provides a bigger SM background. The $f f^{c} H \chi$ coupling also gives rise to new Higgs production mechanism. In any, this sort of novel Higgs physics, especially in the decay to light generation fermions + missing energy, is certainly a smoking gun for our scenario for generating flavor at $\mathrm{TeV}$ energies.

\section{Avoiding large FCNC problems}

If all possible higher dimension operators suppressed by $\sim 1 \mathrm{TeV}$ are present in the SM, there are not only disastrous problems with proton decay but also with flavor-changing effects. The most challenging flavor-changing constraints arise from the Kaon system. If we consider 4 -fermion $\Delta S=2$ operators of the form $C /(1 \mathrm{TeV})^{2} \mathcal{O}$, the bound on the coefficient $\operatorname{Re} C$ from $\Delta m_{K}$ are 13

$$
\begin{aligned}
\mathcal{O}=\left(\bar{d}^{A} \bar{\sigma}^{\mu} s_{A}\right)^{2} & , \quad \operatorname{Re} C<\times 10^{-7} \\
\mathcal{O}=\left(d^{c A} s_{A}\right)^{2} & , \quad \operatorname{Re} C<6 \times 10^{-8} \\
\mathcal{O}=\left(d^{c A} s_{B}\right)\left(d^{c B} s_{A}\right) & , \quad \operatorname{Re} C<3 \times 10^{-7} \\
\mathcal{O}=\left(d^{c A} s_{A}\right)\left(\bar{d}^{B} \overline{s^{c}}{ }_{B}\right) & , \quad \operatorname{Re} C<5 \times 10^{-8} \\
\mathcal{O}=\left(d^{c A} s_{B}\right)\left(\bar{d}^{B} \bar{s}^{c}{ }_{A}\right) & , \quad \operatorname{Re} C<2 \times 10^{-7}
\end{aligned}
$$

where $A, B$ are color indices. The constraints on $\operatorname{Im} C$ from $\epsilon_{K}$ are $\sim 100$ times stronger. Any theory of new physics at the weak weak scale must explain why the coefficients of these operators are so small. Indeed, this is as much a part of the flavor problem as explaining the smallnes of the dimension-4 Yukawa couplings. In this section, we will explore this issue in the context of our mechanism for generating the fermion mass hierarchy.

Before proceeding, let us remember the origin of the problem. In the limit where all Yukawa couplings are set to zero, the SM gauge interactions admit a $U(3)^{5}$ flavor symmetry rotating the three generations of $Q, U^{c}, D^{c}, L, E^{c}$. The three Yukawa couplings matrices $\lambda_{U, D, E}$ explicitly break this $U(3)^{5}$ and are 
the only sources of breaking within the SM. This is reason for the successfully predicted small flavor violation in the SM, the heart of the GIM mechanism. Consider for instance operators of the form

$$
\frac{1}{16 \pi^{2} m_{W}^{2}} \bar{Q}^{a} \bar{\sigma}^{\mu} Q_{b} \bar{Q}^{c} \bar{\sigma}_{\mu} Q_{d} C_{b d}^{a c}
$$

where $a, \cdots, d$ are $U(3)_{Q}$ flavor indices. This is the structure of the operator generated in the usual box diagram. If the only sources of flavor-violation are in the Yukawa matrices $\lambda_{U, D, E}$, purely on gounds of $U(3)^{5}$ transformation properties we must have that

$$
C_{b d}^{a c}=\delta_{c}^{a} \delta_{d}^{b}+\left(\lambda_{U, D}^{\dagger} \lambda_{U, D}\right)_{b}^{a} \delta_{d}^{c}+\left(\lambda_{U, D}^{\dagger} \lambda_{U, D}\right)_{b}^{a}\left(\lambda_{U, D}^{\dagger} \lambda_{U, D}\right)_{d}^{c}+\cdots
$$

where we have omitted overall coefficients in front of each term, and other symmetric terms. For the $\Delta S=2$ operator, we want $C_{12}^{12}$ in the basis where $\lambda_{D}$ is diagonal. The only non-zero contribution arises from

$$
C_{12}^{12} \sim\left[\left(\lambda_{U}^{\dagger} \lambda_{U}\right)_{2}^{1}\right]^{2} \sim \lambda_{c}^{4} \theta_{c}^{2} .
$$

This does not look like the usual GIM suppression factor of $\Delta m_{K}$ which is $\sim\left(m_{c} / m_{W}\right)^{2}$. However, we have to remember that in the usual box diagram, there are light particles running in the loops, and that in fact there is an infrared divergence cutoff by the charm mass which gives an enhancement $\sim$ $1 / \lambda_{c}^{2}$. If physics beyond the SM at the TeV scale has no new sources of flavor violation beyond $\lambda_{U, D, E}$, then there is not even any infrared enhancement of this operator, which then makes negligibly small contributions to $\Delta m_{K}, \epsilon_{K}$. A similar operator analysis can be made to estimate the coefficients of all the other operators in eqn.(26), with the same conclusion. We have learned that as long as the only flavor violation is that given by the Yukawa matrices of the SM, there are no flavor-changing problems with $(\mathrm{TeV})^{-1}$ suppressed operators. This is a well-known fact to flavor model builders (see e.g. [14]). The trouble is that generically, extensions of the SM do have $U(3)^{5}$ violating operators beyond the $\lambda^{\prime}$ s. For instance, in SUSY, we have the 5 scalar mass matrices $m_{Q, U, D, L, E}^{2}$. New invariants in principle unrelated to the Yukawas appear in the coefficients of flavor-violating operators. For instance, the usual squark box diagrams give

$$
C_{12}^{12} \sim\left(\frac{\left(m_{Q}^{2}\right)_{2}^{1}}{4 \pi m_{S U S Y}^{2}}\right)^{2}
$$


with a strong resulting bound on the off-diagonal elements of the squark mass matrices $\sim 10^{-2}-10^{-3}$ depending on the operator. Of course, one could take the attitude that $U(3)^{5}$ is a flavor symmetry broken only by fields whose vevs are the Yukawa matrices [14]. This resolves the flavor-changing problem, but leaves the origin of the hierchies in the Yukawa themselves unexplained. In a four-dimenisonal theory, this question would be relegated to the unknown dynamics of flavor symmetry breaking. As we will see below, in our picture, we can use a $U(3)^{5}$ flavor symmetry to control flavor-changing, while simultaneously offering an understanding of the hierarchies as coming from distant flavor breaking.

We take the flavor symmetry to be $G_{F}=U(3)^{5}$. The idea is to have a different wall generate each non-zero element of the Yukawa matrices $\lambda_{(U, D, E) i^{c}}$. We can then label the walls by the indices $A k l^{c}$, (where $A=U, D, E$ ), a distance $r_{A k l^{c}}$ from our wall. The hypothesis is that on the $\left(A k l^{c}\right)^{\prime}$ th wall, there is a wall-localized field $\phi_{i j^{c}}^{\left(A k l^{c}\right)}$, with the $i, j^{c}$ indices transforming under $G_{F}$ like the Yukawa matrix $\lambda_{A}$. These fields are assumed to have an $\sim M_{s}$ vev of the form

$$
\left\langle\phi_{i j^{c}}^{\left(A k l^{c}\right)}\right\rangle \sim M_{s} \delta_{i}^{k} \delta_{j^{c}}^{l^{c}}
$$

The bulk messengers are $\chi_{A i j^{c}}$ which also transform as $\lambda_{A}$ under $G_{F}$. The interactions responsible for transmitting flavor-breaking to our wall are

$$
\begin{aligned}
\mathcal{L} & \supset \int_{\mathrm{us}} d^{4} x Q^{i} H U^{c j}(x) \chi_{U i j^{c}}(x, y=0)+\text { sim. for D,E } \\
& +\sum_{A k l^{c}} \int_{A k l^{c} \mathrm{Wall}} d^{4} x_{A k l^{c}} \phi_{i j^{c}}^{A k l^{c}} \chi^{* A i j^{c}}\left(x_{A k l^{c}}, y=y_{A k l^{c}}\right)
\end{aligned}
$$

As usual, the sources $\langle\phi\rangle$ will set up a classical profile for $\chi_{A i j^{c}}$,

$$
\left\langle\chi_{A i j^{c}}\right\rangle(x, y)=\sum_{k l^{c}}\left\langle\phi_{i j^{c}}^{A k l^{c}}\right\rangle \Delta_{n}\left(y-y_{A k l^{c}}\right) \sim \Delta_{n}\left(y-y_{A i j^{c}}\right)
$$

where we are once again using units with $M_{s}=1$ and we used the specific form of the vevs in eqn.(31) to get the second equality. The value of $\langle\chi\rangle$ on our wall determines the Yukawa matrices:

$$
\lambda_{A i j^{c}}=\left\langle\chi_{A i j^{c}}\right\rangle(x, y=0) \sim \Delta_{n}\left(r_{A i j^{c}}\right) .
$$

\footnotetext{
${ }^{\dagger}$ Of course, we really mean a sufficiently large discrete subgroup of $U(3)^{5}$, see Section 3.1 .
} 
For massive $\chi$ 's, the inter-wall distances can be within $O(10)$ of the fundamental scale while covering the range of observed Yukawa couplings.

We now wish to show that despite the fact that $U(3)^{5}$ is maximally broken on far away walls, the only flavor violation felt on our wall are given by the "shined" Yukawa matrices $\lambda_{A}$. This is perhaps surprising, since the classical profile of $\chi$ certainly knows about flavor breaking throughout the bulk between our wall and the others where flavor is broken at $O(1)$. However, if the propagation of $\chi$ in the bulk is linear, i.e. if $\chi$ propagates as a free field in the bulk, it is obvious that the only sources of flavor violation in the theory are the vevs $\left\langle\chi_{A}\right\rangle(x, y=0)=\lambda_{A}$. Explicitly, the Lagrangian is of the form

$$
\begin{aligned}
\mathcal{L} & =\int_{\mathrm{US}} d^{4} x Q^{i} U^{c j^{c}} H \chi_{Q i j^{c}}(x, y=0)+\cdots+\text { higher dim. ops. } \\
& +\sum_{A i j^{c}} \int d^{4} x_{A i j^{c}} \operatorname{Tr}\left\langle\chi^{\dagger A}\left(x, y_{A i j^{c}}\right) \phi_{A}\right\rangle \\
& +\int d^{4} x d^{n} y \operatorname{Tr}\left(\partial \chi^{\dagger A} \partial \chi_{A}-m_{\chi_{A}}^{2} \chi^{\dagger A} \chi_{A}\right) .
\end{aligned}
$$

If we now expand $\chi=\langle\chi\rangle+\chi^{\prime}$, we find

$$
\begin{aligned}
\mathcal{L} & =\int_{\mathrm{us}} d^{4} x Q^{i} U^{c j^{c}} H\left(\lambda_{Q i j^{c}}+\chi_{Q i j^{c}}^{\prime}(x, y=0)\right)+\cdots \\
& +\int d^{4} x d^{n} y \operatorname{Tr}\left(\partial \chi^{\prime \dagger A} \partial \chi_{A}^{\prime}-m_{\chi_{A}}^{2} \chi^{\prime \dagger} \chi_{A}^{\prime}\right)
\end{aligned}
$$

The important point is that all the flavor-violating interactions in $\langle\chi\rangle(x, y)$ at points away from $y=0$ have disappeared. All of the higher dimension operators involving SM fields and $\chi$ on our wall then become $U(3)^{5}$ invariants with $\lambda_{A}$ acting as spurions, and we are therefore manifestly safe from FCNC worries induced by the physics above $M_{s}$ even for $M_{s} \sim \mathrm{TeV}$.

This situation changes somewhat if the $\chi^{\prime}$ s have self-interactions in the bulk. As an example, suppose the Lagrangian contains a term of the form

$$
\int d^{4} x d^{n} y h \operatorname{Tr}\left(\chi^{\dagger} \chi\right)^{2}
$$

After expanding $\chi=\langle\chi\rangle+\chi^{\prime}$, we have interactions of the form

$$
\int d^{4} x d^{n} y h \operatorname{Tr}\left(\chi^{\prime \dagger} \chi^{\prime} \chi^{\prime \dagger}\langle\chi\rangle\right)+\cdots
$$


which are now clearly sensitive to the profile of $\langle\chi\rangle$ away from $y=0$. The SM fields can then "sniff" breakings of flavor other than the Yukawas through emitting $\chi$ 's into the bulk which feel $\langle\chi\rangle$ away from $y=0$ through nonlinear interactions. These are not uncontrallably large effects, however. In order to "sniff" a value for $\langle\chi(x, y)\rangle$ significantly different from $\lambda$ which is its value at $y=0$, we must propagate far into the bulk and use the $h$ interaction. However, the propagators to get to this far away point are themselves suppressed.It is a detailed model-builidng question, having to do with the precise configuration of the various walls, whether these effects are harmful. We will not investigate this issue here however, as it is clearly a higher order question. Reiterating: if the $\chi^{\prime} s$ have no self-interactions in the bulk, there is no problem, while the problem is not uncontrollably re-introduced even if there are $O(1)$ self-interactions.

It is evident that more elegant theories of flavor, requiring fewer fields and distant walls, can be constructed. A new and more restrictive framework for flavor model building is suggested in our framework. Given any $G_{F}$ and breaking pattern parametrized by spurions, the small size of the spurions can always be explained by breaking flavor on distant walls. The challenge is to formulate a theory where, remaining fully agnostic of $\mathrm{TeV}$ scale physics and therefore allowing all $G_{F}$ invariant operators suppressed by $(\mathrm{TeV})^{-1}$, flavor changing effects are small enough.

This requirement is stronger than,say, requiring flavor symmetries to solve the SUSY flavor problem. For instance, it is known that a $U(2)$ flavor symmetry acting on all SM fields of the first two generations can solve the SUSY flavor problem by guaranteeing sufficient squark degeneracy [15]. But $U(2)$ fails to satisfy the new criteria. Even before breaking, the following $U(2)$ invariant operator (a,b are $U(2)$ indices)

$$
\left(Q_{a} D_{b}^{c}\right)\left(\bar{Q}^{b} \bar{D}^{c a}\right)
$$

contains a $\Delta S=2$ operator taking e.g. $a=2, b=1$. This therefore requires $M_{s}>1000 \mathrm{TeV}$ to be safe. Why is this operator not generated in the SUSY theory? The reason is that in the limit where $U(2)$ is unbroken, all Yukawa couplings involving the first two generations vanish. The renormalizable interactions of the theory then have an enlarged $U(2)^{5}$ symmetry which forbids $\Delta S=2$ transitions. Operators like the one in eqn.(39), which are are non-zero even before $U(2)$ breaking, can therefore never be 
generated. With SUSY, we are not dealing with an arbitrary theory at $\mathrm{TeV}$ energies. Rather, we have a perturbative, renormalizable QFT, which does not generate all operators consistent with low-energy symmetries. In our case, however, the theory at the $\mathrm{TeV}$ scale is the short distance theory of gravity and is unknown. While it is also possible that in a specific theory not all operators are generated, we can not assume this in any controlled way. We must therefore insist that the group theory and breaking pattern alone suppress dangerous flavor-changing effects. A good starting point may be a $U(2)_{L} \times U(2)_{R}$ symmetry, where the $U(2)_{L}$ acts on the electroweak doublets of the first two generations while the $U(2)_{R}$ acts on the remaining first two generation fields. This retains many of the desirable features of $U(2)$ while forbidding the dangerous $U(2)$ invariant operator we found above. More investigation is however clearly necessary to assess the viability of this model. A theory based on the $S_{3}^{3}$ flavor symmetry is also worth considering [16].

\section{Gauged Symmetries in the bulk}

In [4], the possibility was raised that baryon number is gauged in the bulk. It was shown that if broken only on a wall, the $U(1)_{B}$ gauge boson could naturally get a $\sim(\mathrm{mm})^{-1}$ mass, and could mediate repulsive forces $\sim 10^{6}-10^{8}$ times gravity at sub-mm distances. It was also stated that if $U(1)_{B}$ is broken on a different brane, the proton decay rate can be enormously suppressed. In this section we wish to explore this issue in more detail, for the general case of some arbitrary new gauge symmetry $U(1)_{X}$. Namely, if $U(1)_{X}$ is broken on a different brane by the vev of some field $\phi$ charged under $X$, what level of $X$-violation is generated on our brane?

As we have previously argued, in order to communicate the information of symmetry breaking from one wall to the other, some bulk field must be charged under $U(1)_{X}$. More formally, consider local operaotrs $O_{\text {ourwall }}(x)$ of charge $+q$ and $O_{\text {otherwall }}\left(x^{\prime}\right)$ of charge $-q$. After integrating out all bulk modes, the only gauge invariant operator that can be induced must be of the form

$$
O_{\text {ourwall }}(x) P e^{i q \int_{x, y=0}^{x^{\prime}, y=y_{*}} A d l} O_{\text {otherwall }}\left(x^{\prime}\right)
$$

where the path from $(x, y=0)$ to $\left(x^{\prime}, y=y_{*}\right)$ is unspecified in the path ordered exponential. Since this operator by neccessity involves the gauge 
field in the bulk, the gauge field lines must be able to end in the bulk, i.e. there must be a charged field in the bulk.

Therefore, since the $U(1)_{X}$ gauge boson is neutral under $X$, if the only bulk fields are gravity and $U(1)_{X}$, even after the breaking of $U(1)_{X}$ on the other wall, no $X$ violating operators are induced on our wall. The only possibility to induce $X$ violation is if there are charged modes in the bulk. As we have seen, if these modes are massive $\sim M_{s}$, the coefficient of $X$ violating operators on the wall is exponentially suppressed by $\sim e^{-M_{s} r_{n}}$. If the wall where $U(1)_{X}$ is broken is maximally far away from ours i.e. $r \sim r_{n}$, and if the heavy modes are Planck-scale in mass, the suppression can completely kill proton decay. For $U(1)_{B}$, the relevant proton decay operator would be

$$
\begin{aligned}
\mathcal{L}_{\text {pdecay }} & \sim e^{-\left(M_{s} r_{n}\right)} \frac{Q^{\dagger} U^{c} D^{c} L^{\dagger}}{M_{s}^{2}} \\
& \sim e^{-10^{32 / n}} \frac{Q^{\dagger} U^{c} D^{c} L^{\dagger}}{M_{s}^{2}}
\end{aligned}
$$

giving a lifetimg

$$
\tau_{p} \sim 10^{10^{30 / n}} \times \text { any units }
$$

If there is an additional light messenger field in the bulk carrying $U(1)_{B}$, there are two possible worries. Of course, if this field is lighter than the proton, the proton can decay in a B conserving way with an unsuppressed rate, and so this must be forbidden. Even if the mass of this field is pushed above the proton mass, the exponential suppression of the induced B violating operator may not be sufficient. Indeed, if we ignore the exponential suppression and just use the power suppression for $n>2$, the scale of suppression for the p-decay operator becomes

$$
\mathcal{L}_{\text {pdecay }} \sim \frac{1}{\Lambda^{2}} Q^{\dagger} U^{c} D^{c} L^{\dagger} ; \Lambda=M_{P l} /\left(M_{s} r_{n}\right)
$$

since for a low string scale we need $M_{s} r_{n}$ much larger than 1, the proton would still decay too quickly. Therefore, bulk scalars with unit baryon number must be pushed significantly above the proton mass.

For completeness, we now discuss the possibility that the gauge group $G$ in the bulk is non-abelian. Since the gauge bosons are charged under the gauge group, if $G$ is broken on a different wall, some information of $G$ breaking can be transmitted to our wall. The sort of information is however 
severely constrained again by gauge invariance. The point is that any operator induced on our wall by integrating out the bulk gauge bosons must begin as a gauge invariant operator of the form

$$
O\left(\phi, D_{\mu} \phi\right) f\left(F_{\mu \nu}^{a}\right) \text {. }
$$

When the gauge field lines eminating from this vertex travel to the other wall and feel the breaking of $G$, this can turn into into a $G$ breaking operator $O\left(\phi, \partial_{\mu} \phi\right)$ on the wall. But note that $O$ can not transform under an arbitrary non-trivial represenation of $G$ : it is constrained by the requirement that the above operator be gauge invariant. Only those operators can be generated which contain a singlet in the product with any number of adjoints (contained in $\left.f\left(F_{\mu \nu}^{a}\right)\right)$.

Finally, we note that the bounds from overproduction of light gauge bosons in the bulk in astrophysical systems and in the early universe are similiar to those discussed above for the flavor-conserving interactions of the light $\chi$ fields. For $M_{s} \sim \mathrm{TeV}$, they exclude the cases $n=2,3$; for $n=4$ they require $M_{s}>30 \mathrm{TeV}$, and are safe even with $M_{s} \sim 1 \mathrm{TeV}$ for $n \geq 5$.

\section{Accelerator signals for bulk scalars and vec- tors}

In the previous sections, we have motivated reasons for the existence of scalar and vector fields in the bulk with couplings to wall fields suppressed by the fundamental scale $M_{s}$ : the scalars could be messengers of flavor breaking from distant walls, while the vectors could play a role in stabilizing the proton. For $M_{s} \sim 1 \mathrm{TeV}$ and the case where these fields are very light, there are significant constraints from rare decays, astrophysics and cosmology which force minimally $n>3$. However, if they have masses above a few $\mathrm{GeV}$ all these constraints disappear. Even in this case, however, they can still be produced at colliders (where their small mass is irrelevant compared to the beam energy), much like bulk graviton production [3, 17]. Of course the event must contain a photon or a jet to be visible, so the sort of signal we are interested in are, in complete analogy with the graviton case

$$
\begin{array}{r}
e^{+} e^{-} \rightarrow \gamma+(\text { bulk vector or scalar }=\text { missing energy }) \\
q \bar{q} \rightarrow \text { gluon }+ \text { (bulk vector or scalar }=\text { missing energy })
\end{array}
$$


In fact, just as the long-range forces mediated by these fields are $\sim 10^{6}$ times stronger than gravity, and the astrophysical and cosmological constraints on these fields were stronger than the gravity case, their production cross section at colliders can be comparable to or dominate the graviton production crosssection. Recall that the graviton cross sections at energies $E$ beneath $M_{s}$ scale as

$$
\sigma(\text { grav. prod. }) \sim \frac{E^{n}}{M_{s}^{n+2}}
$$

whereas the scalar and vector cross sections vary as

$$
\begin{gathered}
\sigma(\text { scalar. prod. }) \sim \frac{v^{2} E^{n-2}}{M_{s}^{n+2}} \\
\sigma(\text { vector. prod. }) \sim \frac{E^{n-2}}{M_{s}^{n}}
\end{gathered}
$$

Therefore, at energies beneath $v \sim 200 \mathrm{GeV}$, the scalar production is at least comparable to graviton production, while vector production can dominate for all $E<M_{s}$. Very recent complete analyses for the graviton production at present and future colliders have been carried out in 17] with quite strong results; a similar analysis for bulk scalar and vector production, while more model-dependent, will likely yield a still more powerful probe of $M_{s}$ extending to larger values of $n$.

\section{Electroweak breaking with no Higgs}

In this section we wish to explore another novel possibility raised by the 3-brane universe scenario. Unlike many of the previous observations about flavor, which operate for any value of the fundamental scale $M_{s}$, the considerations of this section, being intimately related to electroweak symmetry breaking, require $M_{s} \sim \mathrm{TeV}$. Up to now, we have rather loosely been referring to the theory on the wall as the "Standard Model", with the tacit assumption of the presence of a light higgs with a negative mass ${ }^{2}$ driving electroweak symmetry breaking. However, this need not be the case. Indeed, as is well known, all the accurately tested aspects SM phenomenology are reproduce by the weakly gauged electroweak chiral Lagrangian. Unitarity breaks down in this theory at energies $\sim 1 \mathrm{TeV}$, and new physics must enter to unitarize it 
at these energies. But there is no reason for a light Higgs to unitarize the theory. Indeed, in our scenario where strong gravity is brought down to the $\mathrm{TeV}$ scale, it is tempting to consider the possibility that physics related to strong gravitational physics at the $\mathrm{TeV}$ scale could trigger dynamical electroweak symmetry breaking. This is not implausible in string theory since it is likely that the theory is neither at weak coupling (since the dilaton runs away), nor at strong coupling (since this is dual to another weakly coupled theory), but at intermediate strong coupling. It is not unreasonable to expect that the theory at intermediate strong coupling may show qualitatively different behavior than that expected from perturbation theory, perhaps including the formation of resonances and dynamical symmetry breaking. We will however explore these ideas within a specific field-theoretic example, "technicolor in the bulk". We will show that even an abelian technicolor group in the bulk can naturally force $\mathrm{TeV}$ condensates for technifermions localised on the wall, triggering electroweak breaking. We can then use ideas from the previous sections for the fermion mass hierarchy, thereby keeping the pleasing picture of technicolor [18] while avoiding the usual problems of Extended Technicolor [19]. The phenomenological purpose of this excercise will be clear: the discovery of e.g. strong $W_{L} W_{L}$ scattering need not imply a usual 4-dimensional technicolor-like theory; it could be the first signal of extra dimensions and strong gravitational effects at the $\mathrm{TeV}$ scale.

As a simple toy example we begin with a theory with Weyl "technifermions" $\Psi, \Psi^{c}$ of charge $+1,-1$ living on our three brane, with a $U(1)_{T C}$ technicolor group living in the $(4+n)$ dimensional bulk. The point is that $U(1)_{T C}$ has a dimensionful interaction strength, which in our case is given by the only short distance scale available, the quantum gravity scale $M_{s} \sim \mathrm{TeV}$ :

$$
g_{T C}^{2}=\frac{h_{T C}^{2}}{M_{s}^{n}}
$$

where $h_{T C}$ is the dimensionless strength of $U(1)_{T C}$ at $M_{s}$. Like gravity itself, $U(1)_{T C}$ becomes strong near the UV cutoff $\sim \mathrm{TeV}$, and the attractive force it mediates between $\Psi, \Psi^{c}$ can trigger the condensate $\left\langle\Psi \Psi^{c}\right\rangle$. The dynamics can be described by an effective Nambu-Jona-Lasinio model, and the condensate will run to the cutoff. This is just what is desired in our case, since the cutoff is at the TeV scale. To understand the physics better, consider the effective 4-fermion operator induced between $\Psi, \Psi^{c}$ from tree-level exchange of the $U(1)_{T C}$ gauge boson. Since $(4+n)$ dimensional momentum is not conseved, 
this involves an integration:

$$
\mathcal{O} \sim \int \frac{d^{n} \kappa}{(2 \pi)^{n}} g_{T C}^{2} \bar{\Psi} \bar{\sigma}^{\mu} \Psi \frac{1}{\kappa^{2}} \bar{\Psi}^{c} \bar{\sigma}_{\mu} \Psi^{c}
$$

For $n>2$, the integral in the above is power divergent in the UV, and must be cutoff at $M_{s} \sim 1 \mathrm{TeV}$. We then obtain a local, attractive four-fermion operator, whici upon Fierzing becomes

$$
\mathcal{O} \sim \frac{h_{T C}^{2}}{(2 \pi)^{n} M_{s}^{2}}\left(\Psi \Psi^{c}\right)\left(\bar{\Psi} \bar{\Psi}^{c}\right)
$$

This is our effective NJL model, which triggers a $\left\langle\Psi \Psi^{c}\right\rangle$ condensate for sufficiently large $h_{T C}$.

It is very easy to extend this analysis to a realistic model. For aesthetic reasons, let the technifermions form one complete SM generation; it is of course possible to choose a smaller technifermion sector. We will choose $U(1)_{T C}$ to be $(B-L)$ for this technifamily. This anomaly-free, and all the $(B-L)$ invariant technifermion bilinears

$$
\left(Q_{T C} U_{T C}^{c}\right),\left(Q_{T C} D_{T C}^{c}\right),\left(L_{T C} E_{T C}^{c}\right)
$$

have the correct Higgs quantum numbers under $S U(2)_{L} \times U(1)_{Y}$, so there is no vacuum alignment problem. Just as in our toy example, $U(1)_{T C}$ becomes strong in the UV and forces a condensate to form. Of course all condensate channels are desirable in our case, however, $\left(L E^{c}\right)$ is the naive most attractive channel and likely condenses first, triggering electroweak breaking. Notice that since we do not need a non-abelian TC group, the technifermion content can be at least twice as small as the usual scenario with the minimal $S U(2)_{T C}$ group, improving the situation with the $S$ parameter. Furthermore, this theory is manifestly non-QCD like, so the usual estimate of the $S$ paramter in QCD-like theories does not apply.

Having used the bulk technicolor for dynamical symmetry breaking, we can use all the ideas of the previous sections for generating small Yukawa couplings by simply replacing $H$ with $\left(\Psi \Psi^{c}\right)_{T C}$. We do not need Extended Technicolor: the theory can still have a flavor symmetry (under which the technifermions may or may not be charged), and the theory above the $\mathrm{TeV}$ scale can naturally generate $\mathrm{TeV}$ suppressed operators linking e.g. $L E^{c}\left(\Psi \Psi^{c}\right)_{T C}$ to the bulk messenger field $\chi$. Of course, a detailed analysis of precision 
electroweak obsevables must be made to assess the viability of this particular model. We do not perform this analysis here. We simply wish to point out that there could be intrinsically higher-dimensional mechansims foe electroweak symmetry breaking without a fundamental Higgs, which in combination with our previous ideas for flavor, can avoid the myriad of FCNC problems associated with Extended Technicolor.

\section{Discussion and conclusions}

Solving the hierarchy problem by bringing the scale of quantum gravity down to the $\mathrm{TeV}$ scale, in the presence of large new spatial dimensions, destroys the desert in short distance scales between the Weak and Planck scales where many mechanisms and phenomena, such as the origin of flavor, neutrino masses and the longevity of the proton, enjoy their usual home. In this paper we have shown that, instead of residing in the ultra-short distances between $10^{-17} \mathrm{~cm}$ and $10^{-33} \mathrm{~cm}$, these phenomena can find a natural home in the large space of the extra dimensions.

Our main focus has been to try to understand the origin of flavor hierarchies at the $\mathrm{TeV}$ scale, while avoiding the usual flavor-changing problems. We accomplish this by supposing that the theory admits a flavor symmetry which prohibits the light generation Yukawa couplings in the flavor symmetric limit. These flavor symmetries are broken with $O(1)$ strength, but on distant branes. This breaking acts as a source which "shines" a bulk scalar field $\chi$, charged under the flavor symmetry. The smallness of the Yukawa couplings follows from the small intensity of the $\chi$ vev shined on our brane, due to the distance between the branes, giving an exponential suppression if $\chi$ is massive, and a power-law suppression if it is (nearly) massless. The origin of the different hierarchies is then reduced to the question of determining the inter-brane separations. We did not address this dynamical problem in detail, but remark that, for exponential or even sufficiently high power suppressions, the branes need not be more separated by more than $O(10)$ times the fundamental length scale in order to span the observed range of Yukawa couplings. The flavor symmetry must be sufficiently powerful to forbid dangerous FCNC operators even after it is broken. We presented an explicit model based on the maximal $U(3)^{5}$ flavor symmetry where essentially the usual GIM mechanism explains the absence of large FCNC effects. 
These ideas for generating small couplings are completely generic and do not depend on a low quantum gravity scale. They can be used to explain small neutrino masses and dynamical SUSY breaking in more conventional theories with a high Planck scale. We also re-iterated that proton longevity can result from gauged baryon number in the bulk, broken on a far away brane. Another interesting possibility for phenomenology from higher dimensions is to do away with a fundamental Higgs on our brane, and trigger electroweak breaking by technicolor dynamics in the bulk. Combined with our other ideas for generating flavor, this can avoid the usual problems of extended technicolor.

There are a number of dramatic experimental signals associated with the various mechanisms suggested in this paper. For any value of the fundamental scale $M_{s}$, if the $\chi$ fields are very light with Compton wavelengths between 1 micron to a millimeter, their exchange gives rise to an attractive, isotope dependent force $10^{6}$ times stronger than gravity at sub-millimeter distances, and can not be missed by the upcoming measurements of submillimeter gravitational strength forces. Light gauge fields in the bulk give rise to similar strength repulsive forces. If the $\chi^{\prime} s$ are heavier than about a $\mathrm{GeV}$ but lighter than physical Higgs particles, they can be produced in novel Higgs decays to light generation fermions $+\chi$, with a width possibly comparable or exceeding the usual $b \bar{b}$ final state. Finally, both bulk gauge fields and $\chi^{\prime} s$ can be produced at colliders, leading to $\gamma+$ missing energy or jet + missing energy signals similar to those from bulk graviton production, with comparable or much larger rates.

\section{Acknowledgements}

It is a pleasure to thank Alex Kagan, Jim Wells and especially Lawrence Hall for valuable discussions. Gia Dvali has informed us of some complementary work addressing flavor physics in the context of theories with low quantum gravity scales [20]. We thank him for discussions. The work of NAH is supported by the Department of Energy under contract DE-AC03-76SF00515. The work of SD is supported in part by NSF grant PHY-9219345-004.

\section{References}


[1] H. Georgi and S.L. Glashow, Phys. Rev. Lett. 32 (1974) 438.

[2] N. Arkani-Hamed, S. Dimopoulos and G. Dvali, Phys. Lett. B431 (1998) 329.

[3] I. Antoniadis, N. Arkani-Hamed, S. Dimopoulos and G. Dvali, hepph/9804398.

[4] N. Arkani-Hamed, S. Dimopoulos and G. Dvali, hep-ph/9807344.

[5] R. Sundrum, hep-ph/9805471.

[6] N. Arkani-Hamed, S. Dimopoulos and J. March-Russell, hepth/9809124.

[7] J.C. Long, H.W. Chan and J.C. Price, hep-ph/9805217.

[8] C.D.Froggatt and H.B. Nielsen, Nucl. Phys. B147 (1979) 277.

[9] S. Dimopoulos and G. Giudice, Phys. Lett. B379 (1996) 105.

[10] N. Arkani-Hamed, S. Dimopoulos, G. Dvali and J. March-Russell, in preparation.

[11] P. Brax and N. Turok, Phys. Lett. B413 (1997) 331.

[12] E.A. Mirabelli and M.E. Peskin, hep-th/9712214.

[13] See e.g. Nima Arkani-Hamed, C.D. Carone, L.J. Hall and H.Murayama, Phys. Rev. D54 (1996) 7032.

[14] L.J. Hall and L. Randall, Phys. Rev. Lett. 65 (1990) 2939.

[15] R. Barbieri, G. Dvali and L.J. Hall, Phys. Lett. B377 (1996) 76.

[16] L.J. Hall and H. Murayama, Phys. Rev. Lett. 75 (1995) 3985.

[17] G.F. Giudice, R. Rattazzi and J.D. Wells, hep-ph/9811291; E. Mirabelli, M. Perlestein and M. Peskin, to appear; J.L. Hewett, to appear.

[18] L. Susskind, Phys. Rev. D20 (1979) 2619.

[19] S. Dimopoulos and L. Susskind, Nucl. Phys. B155 (1979) 237. 
[20] Z. Berezhiani and G. Dvali, in preparation. 